\title{
Lung ultrasound in the management of acute heart failure
}

\author{
yanmei zhang ${ }^{1}$, hai $\mathrm{xu}^{1}$, xiaochen wang $^{1}$, and banglong $\mathrm{xu}^{1}$
}

${ }^{1}$ Affiliation not available

June 16, 2020

\begin{abstract}
Acute heart failure (AHF) is a common clinical emergency characterized by pulmonary edema, with rapid progression and poor prognosis. In the past, clinicians mostly used physical examination, chest radiography (CXR) and brain-type natriuretic peptide (BNP) for diagnosis and evaluation of the treatment effects of AHF, but their application is limited due to the long duration and low sensitivity and specificity. Recently, lung ultrasound (LUS) is found to be a simple, fast and effective approach to detect pulmonary edema and diagnose AHF. Here we aimed to provide a review on the use of LUS in management of AHF, which focused on the application of LUS in diagnosis, treatment and prognosis of AHF
\end{abstract}

\section{Hosted file}

lus manuscript.doc available at https://authorea.com/users/334032/articles/460104-lungultrasound-in-the-management-of-acute-heart-failure 